\title{
Polarized gluon TMDs at small $x$
}

\author{
Piet J. Mulders*t \\ Nikhef Theory Group and VU, Amsterdam \\ E-mail: p.j.g.muldersevu.nI

\section{Elena Petreska} \\ Nikhef Theory Group and VU, Amsterdam \\ E-mail: petreska@nikhef.nI
}

\begin{abstract}
Flavor, spin and partonic transverse momenta are important characteristics for parton distribution functions (PDFs), allowing a proliferation of possibilities. This proliferation can provide novel information into the non-perturbative structure of nucleons as well as new ways of probing high energy processes. Wilson lines are an important ingredient in the operator definition of transverse momentum dependent PDFs (TMDs). We focus on small $\mathrm{x}$ behavior of unpolarized and linearly polarized gluon TMDs, in particular those with a dipole gauge link structure, for unpolarized and transversely polarized nucleons. For this we employ generalized TMD correlators (GTMDs) involving non-forward matrix elements of Wilson loops. As an example of the richness of GTMDs, we note that the C-odd parts can generate odd harmonics in the two-particle azimuthal correlations in peripheral proton-nucleus collisions.
\end{abstract}

XXVII International Workshop on Deep-Inelastic Scattering and Related Subjects - DIS2019 8-12 April, 2019

Torino, Italy

\footnotetext{
* Speaker.

${ }^{\dagger}$ details can be found in Ref. [1]
} 


\section{Introduction}

High energy processes provide possibilities to access the quark and gluon structure of nucleons via collinear parton distribution functions (PDFs) depending on collinear momentum fraction $x$ or transverse momentum dependent PDFs (TMDs) depending on $x$ and $p_{T}$. As nonperturbative objects these functions appear in the parametrization of matrix elements of nonlocal field configuration. For instance for processes involving gluons the naive polarization sum is to be replaced by a lightfront (LF) correlator

$$
\begin{aligned}
& \varepsilon^{\mu}(k) \varepsilon^{\nu *}(k) \rightarrow \Gamma^{\left[U, U^{\prime}\right] \mu v}\left(x, k_{T} ; n\right) \\
& \Gamma^{\left[U, U^{\prime}\right] \mu v}\left(x, k_{T} ; n\right)=\left.\int \frac{d z \cdot P d^{2} z_{T}}{(2 \pi)^{3}} e^{i k \cdot z}\left\langle P\left|F^{n \mu}(0) U_{[0, z]} F^{n v}(z) U_{[z, 0]}^{\prime}\right| P\right\rangle\right|_{\mathrm{LF}}
\end{aligned}
$$

where $x=k \cdot n$ and LF implies $z \cdot n=0$. The nonlocal matrix elements require the presence of gauge links, which in the case of TMDs can be staple-like gauge links with Wilson lines running along the usual light-like vector $n$ characterizing the incoming hadron in the high-energy process. One of the fundamental gluon TMD distributions, usually referred to as dipole distribution, involves one staple-like gauge link running to plus infinity and one running to minus infinity. This gauge structure can be related to a simple Wilson loop with a transverse separation. Before addressing gluon TMDs in unpolarized and polarized hadrons, we will first recall the emergence of Wilson loops in the case of generalized transverse momentum dependent distributions (GTMDs), and how TMDs can be obtained in the forward limit of GTMDs.

\section{Gluon GTMDs and the Wilson loop}

GTMD correlators of partons inside hadrons are off-forward matrix elements that combine information on TMDs and generalized parton distributions (GPDs). The dipole GTMD involves "+" and "-" gauge links (using $d^{3} z=d z \cdot P d^{2} z_{T}$ )

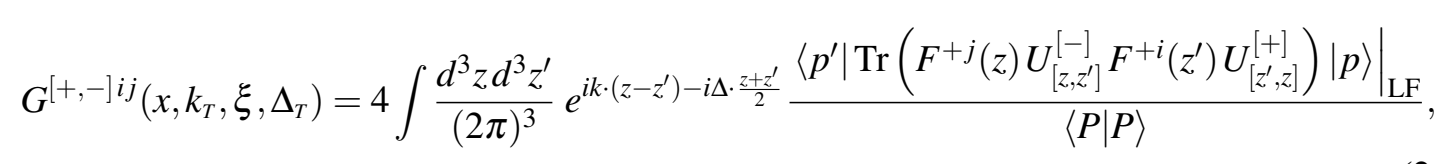

where the average hadron momentum is given by $P \equiv\left(p^{\prime}+p\right) / 2$, the momentum transfer by $\Delta \equiv$ $p^{\prime}-p$, the average gluon momentum is denoted by $k, x$ is the longitudinal momentum fraction and $\xi=-\Delta \cdot n / 2$ is the skewness parameter. We are interested in the limit of small $x$ when the gluonic content of the hadron is the most relevant. In the limit of $x$ and $\xi$ going to zero, the minus integration can be expressed as a gluonic pole operator,

$$
G_{T}^{\alpha}(x) \equiv \frac{1}{2} \int_{-\infty}^{\infty} d \eta^{-} U_{\left[x^{-}, \eta^{-} ; \mathbf{x}\right]}^{n} F^{+\alpha}\left(x^{+}, \eta^{-}, \mathbf{x}\right) U_{\left[\eta^{-}, x^{-} ; \mathbf{x}\right]}^{n},
$$

and upon using the relation [2]

$$
\left[i \partial_{\mathbf{x}}^{\alpha}, U_{[a, x]}^{[ \pm]}\right]= \pm g U_{[a, x]}^{[ \pm]} G_{T}^{\alpha}(x)
$$


the dipole GTMD can be written simply in terms of a Wilson loop gauge link, $U^{[\square]}[1]$ :

$$
G^{[+,-] i j}\left(k_{T}, \Delta_{T}\right)=\frac{2 N_{c}}{\alpha_{s}}\left[\frac{1}{2}\left(k_{T}^{2}-\frac{\Delta_{T}^{2}}{4}\right) g_{T}^{i j}+k_{T}^{i j}-\frac{\Delta_{T}^{i j}}{4}-\frac{k_{T}^{[i} \Delta_{T}^{j]}}{2}\right] G^{[\square]}\left(k_{T}, \Delta_{T}\right),
$$

where

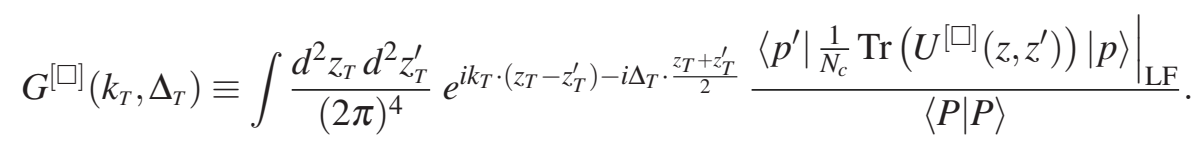

The GTMD correlator can be parametrized in terms of various gluon GTMD distribution functions, which in the case of the Wilson loop correlator above reduce to a single function and we choose the following parametrization:

$$
G^{[\square]}\left(k_{T}, \Delta_{T}\right)=\frac{\alpha_{s}}{2 N_{c} M^{2}} \mathscr{E}\left(k_{T}^{2}, \Delta_{T}^{2}, k_{T} \cdot \Delta_{T}\right),
$$

where $\mathscr{E}$ is a Wilson loop GTMD that in the forward limit gives $\lim _{\Delta \rightarrow 0} \mathscr{E}\left(k_{T}^{2}, \Delta_{T}^{2}, k_{T} \cdot \Delta_{T}\right)=e\left(k_{T}^{2}\right)$, with $e\left(k_{T}^{2}\right)$ the Wilson loop TMD from Ref. [3].

\section{Gluon TMDs in unpolarized hadrons}

Returning to the gluon correlator in Eq. 1.2 one can use its standard parametrization for an unpolarized hadron $[4,5]$,

$$
\Gamma^{\left[U, U^{\prime}\right] i j}\left(x, k_{T}\right)=\frac{x}{2}\left\{-g_{T}^{i j} f_{1}^{\left[U, U^{\prime}\right]}\left(x, k_{T}^{2}\right)+\frac{k_{T}^{i j}}{M^{2}} h_{1}^{\perp\left[U, U^{\prime}\right]}\left(x, k_{T}^{2}\right)\right\},
$$

The GTMD treatment in the previous paragraph shows the small $\mathrm{x}$ behavior of the gluon TMDs,

$$
\begin{aligned}
& \lim _{x \rightarrow 0} f_{1}^{[+,-]}\left(x, k_{T}^{2}\right)=\frac{k_{T}^{2}}{2 M^{2}} e^{[+,-]}\left(x, k_{T}^{2}\right), \\
& \lim _{x \rightarrow 0} h_{1}^{\perp[+,-]}\left(x, k_{T}^{2}\right)=e^{[+,-]}\left(x, k_{T}^{2}\right),
\end{aligned}
$$

which shows that the importance of the gluon dipole TMDs at small $\mathrm{x}$, and their expected complete linear polarization reaching the maximal bound for $h_{1}^{\perp}$.

\section{Gluon TMDs in polarized hadrons}

With the forward extension of Eq. 2.6 to

$$
\lim _{\Delta \rightarrow 0} G^{[\square]}\left(k_{T}, \Delta_{T}\right)=\frac{1}{2 M^{2}}\left\{e\left(k_{T}^{2}\right)-\frac{\varepsilon_{T}^{k S_{T}}}{M} e_{T}\left(k_{T}^{2}\right)\right\},
$$

and the standard parametrizations for longitudinally and transversely polarized nucleons,

$$
\begin{aligned}
\Gamma_{L}^{i j\left[U, U^{\prime}\right]}\left(x, k_{T}\right)= & \frac{x}{2}\left\{i \varepsilon_{T}^{i j} S_{L} g_{1}^{\left[U, U^{\prime}\right]}\left(x, k_{T}^{2}\right)+\frac{\varepsilon_{T \alpha}^{\{i} k_{T}^{j\} \alpha}}{M^{2}} S_{L} h_{1 L}^{\perp\left[U, U^{\prime}\right]}\left(x, k_{T}^{2}\right)\right\} \\
\Gamma_{T}^{i j\left[U, U^{\prime}\right]}\left(x, k_{T}\right)= & \frac{x}{2}\left\{\frac{g_{T}^{i j} \varepsilon_{T}^{k S_{T}}}{M} f_{1 T}^{\perp\left[U, U^{\prime}\right]}\left(x, k_{T}^{2}\right)-\frac{i \varepsilon_{T}^{i j} k_{T} \cdot S_{T}}{M} g_{1 T}^{\left[U, U^{\prime}\right]}\left(x, k_{T}^{2}\right)\right. \\
& \left.-\frac{\varepsilon_{T}^{k\{i} S_{T}^{j\}}+\varepsilon_{T}^{S_{T}\{i} k_{T}^{j\}}}{4 M} h_{1}^{\left[U, U^{\prime}\right]}\left(x, k_{T}^{2}\right)-\frac{\varepsilon_{T \alpha}^{\{i} k_{T}^{j\} \alpha S_{T}}}{2 M^{3}} h_{1 T}^{\perp\left[U, U^{\prime}\right]}\left(x, k_{T}^{2}\right)\right\}
\end{aligned}
$$


we obtain the small $x$ limit of polarized dipole-type TMDs, which are zero for the TMDs in a longitudinally polarized nucleon and

$$
\begin{aligned}
& \lim _{x \rightarrow 0} f_{1 T}^{\perp[+,-]}\left(x, k_{T}^{2}\right)=\frac{k_{T}^{2}}{2 M^{2}} e_{T}^{[+,-]}\left(x, k_{T}^{2}\right), \\
& \lim _{x \rightarrow 0} h_{1}^{[+,-]}\left(x, k_{T}^{2}\right)=\frac{k_{T}^{2}}{2 M^{2}} e_{T}^{[+,-]}\left(x, k_{T}^{2}\right), \\
& \lim _{x \rightarrow 0} h_{1 T}^{\perp[+,-]}\left(x, k_{T}^{2}\right)=e_{T}^{[+,-]}\left(x, k_{T}^{2}\right),
\end{aligned}
$$

which shows that the TMDs in transversely polarized nucleon approach a common function $e_{T}$, which itself is bounded by $e$ via $\left(\left|k_{T}\right| / M\right) e_{T}\left(k_{T}^{2}\right) \leq e\left(k_{T}^{2}\right)[6]$

\section{Nuclear measurements of gluon GTMDs}

In this section we extend the study of unpolarized gluon GTMDs to the nuclear case. In particular, we will study the C-odd dipole gluon GTMD in a nuclear environment, which can be related to the odderon operator $\mathscr{O}\left(z, z^{\prime}\right) \equiv 1 / 2 i N_{c} \operatorname{tr}\left(U^{[\square]}-U^{[\square] \dagger}\right)$ in the small-x limit,

$$
\begin{aligned}
G_{(d)}^{(\mathrm{T}-\text { odd })}\left(k_{T}, \Delta_{T}\right) & \equiv \frac{1}{2}\left(G^{[+,-]}\left(k_{T}, \Delta_{T}\right)-G^{[-,+]}\left(k_{T}, \Delta_{T}\right)\right) \\
& =\frac{2 i N_{c}}{\alpha_{s}} \int \frac{b_{T} d^{2} r_{T}}{(2 \pi)^{4}} e^{i k_{T} \cdot r_{T}-i \Delta_{T} \cdot b_{T}}\left\langle\mathscr{O}\left(b_{T}+\frac{r_{T}}{2}, b_{T}-\frac{r_{T}}{2}\right)\right\rangle
\end{aligned}
$$

From the hermiticity and time reversal constraints of this correlator it follows that the odderon can generate only odd harmonics $\cos \left[(2 n-1)\left(\phi_{k}-\phi_{\Delta}\right)\right]$, with $n \geq 1$. In order to access these odd harmonics in high-energy collisions, we will use the dipole Wigner distribution, obtained from a Fourier transform of the GTMD, $W^{[\square]}\left(b_{T}, k_{T}\right)=\int d^{2} \Delta_{T} e^{i \Delta_{T} \cdot b_{T}} G^{[\square]}\left(k_{T}, \Delta_{T}\right)$. For an individual nucleon, and for an infinite nucleus with constant nucleon density, the Wigner distribution is independent of the impact parameter $\mathbf{b}$ when $\Delta=0$. However, for a realistic finite nucleus with some non-uniform transverse nuclear profile, the Wigner distribution depends on the impact parameter even if $\Delta \approx 0$ for the nucleons inside the nucleus. In a large nucleus the dipole Wigner distribution is then related to [1]

$$
W_{A}^{[\square]}\left(b_{T}, k_{T}\right)=\int \frac{d^{2} r_{T}}{(2 \pi)^{2}} e^{i k_{T} \cdot r_{T}}\left\langle\frac{1}{N_{c}} \operatorname{tr} U^{[\square]}\left(b_{T}+\frac{r_{T}}{2}, b_{T}-\frac{r_{T}}{2}\right)\right\rangle_{A},
$$

where the nuclear averaged matrix element $\langle\ldots\rangle_{A}$ is evaluated between nucleon states at a given impact parameter $b_{T}$ in the nucleus.

We consider the production of two hadrons at forward rapidity in peripheral pA collisions, from double parton scattering. The final state hadrons are produced by two quarks from the proton's wavefunction, after scattering off the nucleus, which, in this particular phenomenological setup, is in a state of high-gluon densities [7]. Therefore, this process is sensitive to saturation effects in the nucleus, and we use the Color Glass Condensate (CGC) approach [8] to describe its wavefunction. The two quarks scatter independently and each acquires a phase described with a fundamental Wilson line, which on the level of the cross section, lead to a product of two dipoles 
$\left\langle S\left(\mathbf{x}_{1}, \mathbf{y}_{1}\right) S\left(\mathbf{x}_{2}, \mathbf{y}_{2}\right)\right\rangle_{x, A}$ averaged over the CGC state of the nucleus. In the large- $N_{c}$ limit the scattering of the two quarks can be factorized, and the differential cross section can be written in terms of the individual Wigner distributions:

$$
\frac{d \sigma^{p A}}{d y_{1} d y_{2} d^{2} k_{1 T} d^{2} k_{2 T}} \propto \int d^{2} b_{1 T} d^{2} b_{2 T} F_{p}\left(x_{1}, x_{2}, b_{1 T}-b_{2 T}\right) x W\left(x, b_{1 T}, k_{1 T}\right) x W\left(x, b_{2 T}, k_{2 T}\right),
$$

where $F_{p}\left(x_{1}, x_{2}, b_{T 1}-b_{T 2}\right)$ describes the double parton distribution in the proton. To extract the angular correlations between the impact parameter and the transverse momentum of the particles, we parametrize the Wigner distribution in terms of the different harmonic contributions [9]:

$$
\begin{array}{r}
x W\left(x, b_{T}, k_{T}\right)=x \mathscr{W}_{0}\left(x, b_{T}^{2}, k_{T}^{2}\right)+2 \cos \left(\phi_{b}-\phi_{k}\right) x \mathscr{W}_{1}\left(x, b_{T}^{2}, k_{T}^{2}\right) \\
+2 \cos 2\left(\phi_{b}-\phi_{k}\right) x \mathscr{W}_{2}\left(x, b_{T}^{2}, k_{T}^{2}\right)+\ldots
\end{array}
$$

We are interested in studying the first odd azimuthal particle correlation in this process, which will clearly be connected to $\mathscr{W}_{1}$, what we call the odderon Wigner distribution. The way of quantifying these correlations is through the flow coefficients $v_{n}\left(k_{T}, k_{T}{ }^{\text {ref }}\right) \equiv V_{n}\left(k_{T}, k_{T}{ }^{\text {ref }}\right) / \sqrt{V_{n}\left(k_{T}{ }^{\text {ref }}, k_{T}{ }^{\text {ref }}\right)}$. For the first odd flow coefficient we get [1] (using a Gaussian ansatz for the transverse density profile of the proton):

$$
V_{1}\left(k_{1 T}^{2}, k_{2 T}^{2}\right) \equiv \frac{\int d b_{1 T}^{2} d b_{2 T}^{2} e^{-\frac{b_{1 T}^{2}+b_{2}^{2}}{4 R_{N}^{2}}} I_{1}\left(\frac{b_{1 T} b_{2 T}}{2 R_{N}^{2}}\right) x \mathscr{W}_{1}\left(x, b_{1 T}^{2}, k_{1 T}^{2}\right) x \mathscr{W}_{1}\left(x, b_{2 T}^{2}, k_{2 T}^{2}\right)}{\int d b_{1 T}^{2} d b_{2 T}^{2} e^{-\frac{b_{1 t}^{2}+b_{2 T}^{2}}{4 R_{N}^{2}}} I_{0}\left(\frac{b_{1 T} b_{2 T}}{2 R_{N}^{2}}\right) x \mathscr{W}_{0}\left(x, b_{1 T}^{2}, k_{1 T}^{2}\right) x \mathscr{W}_{0}\left(x, b_{2 T}^{2}, k_{2 T}^{2}\right)} .
$$

With the above analysis we showed that the C-odd gluon Wigner distribution can serve as a source for non-zero odd particle correlations in pA collisions, stemming only from the initial correlations in the incoming nucleus. Non-zero odd correlations have been observed experimentally in pA collisions at RHIC and LHC; here we compare our findings to the experimental data from the ATLAS collaboration [10]. For this purpose, we use particular models for $\mathscr{W}_{0}$ and $\mathscr{W}_{1}$. Namely, $\mathscr{W}_{0}$ is connected to the pomeron operator, which we evaluate in the McLerranVenugopalan model [11], while $\mathscr{W}_{1}$ is related to the odderon operator, for which we use the result from Ref. [12]. Both of these functions involve the saturation scale $Q_{s}^{2}(b)$ characterizing the nonlinear gluon dynamics in the nucleus, which in turn is connected to the nuclear profile function $T(b)$, $Q_{s}^{2}(b) \equiv 4 \pi \alpha_{s}^{2} C_{F} / N_{c} T(b)$. Finally, for $T(b)$ we use the Woods-Saxon distribution. We perform an approximate numerical calculation (without any fit parameters) and we find that the magnitude of $v_{1}$ for lower values of $\mathrm{k}$ is of the same order as the maximal value observed in the data [1]. We plot our results for a lead nucleus with $A=208$ and for a copper nucleus with $A=63$ in Fig. 1.

In summary, we showed that odd azimuthal two-particle correlations in pA collisions can arise without breaking of the rotational symmetry of the target, but only from its radial profile, and at leading order in $1 / N_{c}$. The source of the odd harmonics is the C-odd part of the nuclear Wigner distribution and the correlations between the impact parameter of the particle and its transverse momentum. The effect is the largest at the edge of the nucleus, i.e. for peripheral collisions. 


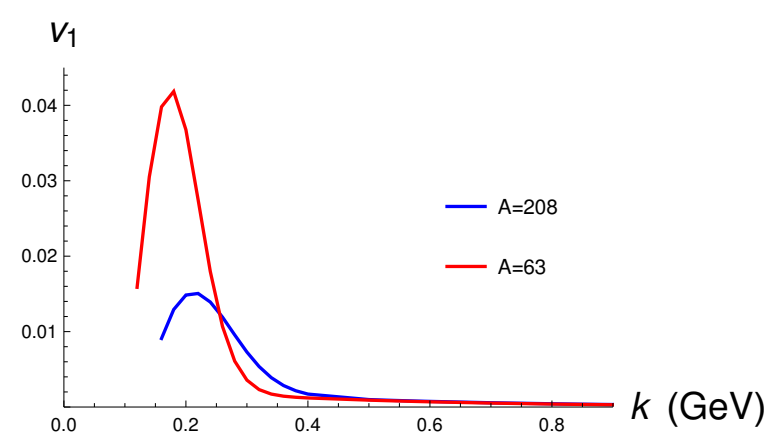

Figure 1: The directed flow $v_{1}(k)$ for a lead nucleus with $A=208$ (blue curve) and for a copper nucleus with $A=63$ (red curve). We take $\alpha_{s}=0.3, \Lambda=0.24 \mathrm{GeV}$, and $k^{\text {ref }}=0.8 \mathrm{GeV}$.

\section{References}

[1] D. Boer, T. Van Daal, P. J. Mulders and E. Petreska, JHEP 1807, 140 (2018), doi:10.1007/JHEP07(2018)140 [arXiv:1805.05219 [hep-ph]].

[2] M. G. A. Buffing, A. Mukherjee and P. J. Mulders, Phys. Rev. D 88, 054027 (2013), doi:10.1103/PhysRevD.88.054027 [arXiv:1306.5897 [hep-ph]].

[3] D. Boer, S. Cotogno, T. van Daal, P. J. Mulders, A. Signori and Y. J. Zhou, JHEP 1610, 013 (2016), doi:10.1007/JHEP10(2016)013 [arXiv:1607.01654 [hep-ph]].

[4] P. J. Mulders, and J. Rodrigues, Phys. Rev. D63, 094021 (2001), doi:10.1103/PhysRevD.63.094021 [hep-ph/0009343].

[5] S. Meissner, A. Metz and K. Goeke, Phys. Rev. D76, 034002 (2007), doi:10.1103/PhysRevD.76.034002 [hep-ph/0703176].

[6] S . Cotogno, T. van Daal and P. J. Mulders, JHEP 11, 185 (2017), doi:10.1007/JHEP11(2017)185 [arXiv:1709.07827 [hep-ph]].

[7] A. Dumitru, A. Hayashigaki and J. Jalilian-Marian, Nucl. Phys. A 765, 464 (2006), doi:10.1016/j.nuclphysa.2005.11.014 [hep-ph/0506308].

[8] F. Gelis, E. Iancu, J. Jalilian-Marian and R. Venugopalan, Ann. Rev. Nucl. Part. Sci. 60, 463 (2010), doi:10.1146/annurev.nucl.010909.083629 [arXiv:1002.0333 [hep-ph]].

[9] Y. Hatta, B. W. Xiao and F. Yuan, Phys. Rev. Lett. 116, no. 20, 202301 (2016), doi:10.1103/PhysRevLett.116.202301 [arXiv:1601.01585 [hep-ph]].

[10] G. Aad et al. [ATLAS Collaboration], Phys. Rev. C 90, no. 4, 044906 (2014), doi:10.1103/PhysRevC.90.044906 [arXiv:1409.1792 [hep-ex]].

[11] L. D. McLerran and R. Venugopalan, Phys. Rev. D 49, 2233 (1994), Phys. Rev. D 49, 3352 (1994);

[12] Y. V. Kovchegov and M. D. Sievert, Phys. Rev. D 86, 034028 (2012), Erratum: [Phys. Rev. D 86, 079906 (2012)], doi:10.1103/PhysRevD.86.034028, 10.1103/PhysRevD.86.079906 [arXiv:1201.5890 [hep-ph]]. 